Research Article

\title{
First-Passage Time Model Driven by Lévy Process for Pricing CoCos
}

\author{
Xiaoshan Su and Manying Bai \\ Department of Finance, Beihang University, Beijing 100191, China \\ Correspondence should be addressed to Xiaoshan Su; suxiaoshan@buaa.edu.cn
}

Received 26 July 2016; Accepted 12 December 2016; Published 11 January 2017

Academic Editor: Jean J. Loiseau

Copyright (C) 2017 X. Su and M. Bai. This is an open access article distributed under the Creative Commons Attribution License, which permits unrestricted use, distribution, and reproduction in any medium, provided the original work is properly cited.

\begin{abstract}
Contingent convertible bonds (CoCos) are typical form of contingent capital that converts into equity of issuing firm or writes down if a prespecified trigger occurs. This paper proposes a general Lévy framework for pricing CoCos. The Lévy framework indicates that the difficulty in giving closed-form expression for CoCos price is the possible introduction of the Lévy process whose firstpassage time problem has not been solved. According to characteristics of new Lévy measure after the measure transform, three specific Lévy models driven by drifted Brownian motion, spectrally negative Lévy process, and double exponential jump diffusion process are proposed to give the solution keeping the form of the driving process unchanged under the measure transform. These three Lévy models provide closed-form expressions for CoCos price while the latter two possess them up to Laplace transform, whose pricing results are given by combining with numerical Fourier inversion and Laplace inversion. Numerical results show that negative jumps have large influence on CoCos pricing and the Black-Scholes model would overestimate CoCos price by simply compressing jumps information into volatility while the other two models would give more accurate CoCos price by taking jump risk into consideration.
\end{abstract}

\section{Introduction}

Contingent convertible bonds (CoCos) are typical form of contingent capital that converts into equity of issuing firm or writes down if a prespecified trigger occurs. This financial product can timely and efficiently improve capital structure of the issuing firm through transforming debt burden into equity capital on balance sheet when the issuing firm faces financial distress. In the financial crisis of 2007-2009, many banks in distress are rescued by their governments with lots of taxpayers' money, which causes loud public criticism. To prevent government bailouts in the future, the regulatory institutions in many countries propose that market means should be employed to solve too-big-to-fail problem and advocate banks to establish emergency mechanism to improve their loss absorption capacities. Since CoCos have strong capacity of loss absorption, issuing CoCos becomes a good scheme for establishing emergency mechanism of banks. For the whole financial system, CoCos can play the role of capital buffer to enhance stability of the whole financial system. Basel III recommends CoCos as an important instrument of banks capital management and emphasizes that banks should hold adequate CoCos reserve to cope with possible crisis. To satisfy the requirement of risk management in Basel III, many banks have issued CoCos. Since Lloyds Banking Group first issued CoCos in size of 7 bn GBP in December 2009, many banks, including Rabobank, Credit Suisse, Bank of Cyprus, UBS, and Macquarie, have issued CoCos. According to the investigation of IMF, America, England, Canada, and Netherland all have the intention to implement CoCos into the regulation system. The S\&P [1] estimates that the overall potential issuance of CoCos would amount to at least 1 trillion dollars.

Flannery (2003) [2] proposes early form of CoCos called reserve convertible debentures (RCD) to address too-bigto-fail problem. Flannery (2010) [3] updates his proposal and renames it as contingent capital certificates. Squam Lake Working Group (2009) [4] proposes CoCos with a dual trigger based on regulatory and accounting values. McDonald (2013) [5] also proposes a dual trigger while the trigger is based on market values including the issuing firm's stock price and a financial institutions index. These two designs of CoCos 
with a dual trigger can guarantee that issuing firm is only protected during financial crisis. The trigger mechanism is the key design for CoCos. So far accounting trigger, market trigger, regulatory trigger, and their combination multivariate trigger have been proposed. Most issued CoCos have an accounting trigger and some have an additional regulatory trigger; there are some arguments against accounting triggers involving accounting manipulation and discontinuous monitoring. Market trigger is advocated to use since market information is updated continuously and transparently. Though market trigger could also potentially be manipulated to trigger conversion, the market manipulations could be more easily and timely observed and intervened by regulation institutions. However, hedging equity exposure by taking short position in the underlying shares would result in the death-spiral effect. In addition, Sundaresan and Wang (2015) [6] point out that CoCos with a simple market trigger would lead to multiplicity or absence of an equilibrium. Calomiris and Herring (2013) [7] suggest using a market trigger based on a 90-day moving average of the ratio of the market value of equity to the sum of the market value of equity and the face value of debt and prove that this design could preserve a unique equilibrium. Pennacchi et al. (2014) [8] propose to add buy-back clause for the converted shares into CoCos to avoid problems of CoCos with market-based triggers such as market manipulation. Corcuera et al. (2014) [9] analyze Coupon Cancellable CoCos where coupons can be cancelled due to their own triggers to alleviate the death-spiral effect. In trigger mechanism, there are different conversion ways and previous designs of CoCos are all-at-once conversion except Flannery's (2003) [2] original proposal. Glasserman and Nouri (2012) [10] analyze the partial and ongoing conversion of CoCos with an accounting trigger. Different designs of CoCos take their own advantages, but the designs are far from perfect and the arguments about the design of CoCos are still going on.

The design of CoCos leads to the work on valuation. The main proposed CoCos pricing models include intensity model and first-passage time model. The intensity model is also named as credit derivatives model in some papers and the first-passage time model can be regarded as consisting of structural model and equity derivatives model since they all use barrier approach based on first-passage time distribution. The intensity model has been studied by De Spiegeleer and Schoutens (2012) [11] and Cheridito and Xu (2015) [12]. However, most studies concentrate on the first-passage time model. In the first-passage time model, the trigger process can be either an accounting ratio or a market price. Several papers employ structural models to obtain CoCos price, but their focuses are more on CoCos role and behaviours. Pennacchi (2010) [13] investigates the influence of contractual terms and different risk source of issuing firm on the value of CoCos by simulation in a jump diffusion structural model. Koziol and Lawrenz (2012) [14] show that CoCos would distort risk taking incentives and possibly create negative externalities under some conditions. Berg and Kaserer (2015) [15] show that CoCos would exacerbate risk taking incentives of shareholders and debt overhang problem. Hilscher and Raviv (2014) [16] point out that banks issuing CoCos would have a lower default probability and appropriate design of CoCos could entirely eliminate risk-shifting incentives of shareholders. Himmelberg et al. (2014) [17] show that CoCos can, if properly designed, make banks have an incentive to pursue conservative capital structures to avoid dilution risk from forced conversion and mitigate debt overhang problem. Albul et al. (2015) [18] use structural model to determine the CoCos role in optimal capital structure of issuing firm in an infinite maturity setting and analyze CoCos behaviours in different scenes. Metzler and Reesor (2015) [19] indicate that terms of conversion fundamentally alter the nature of CoCos through Merton-type structural model. Some more theoretical works study equity derivatives models and Wilkens and Bethke (2014) [20] show that the equity derivatives model is the most practical model for pricing and risk management of CoCos by comparing the credit derivatives model, the structural model, and the equity derivatives model through empirical study based upon largely fitting market price. De Spiegeleer and Schoutens (2012) [11] approximate the event breaching noncontinuously observable accounting trigger level by an event where continuously observable stock price process falls under an implied stock price barrier and they model the issuing firm's stock price process as a geometric Brownian motion and derive closed-form expression for CoCos price. Cheridito and Xu (2015) [12] develop a general continuous model and obtain CoCos price by solving parabolic partial differential equation with Dirichlet boundary conditions. These equity derivatives models assume that the trigger process moves in a continuous manner, which amounts to neglecting the abrupt movements in which most of the risk is concentrated. The discontinuous models describing jump risk from exogenous shock worth studying and De Spiegeleer and Schoutens (2012) [11] also suggest their Black-Scholes model to be extended to the model driven by Lévy process to incorporate jumps and heavy tails to improve accuracy for CoCos pricing. Corcuera et al. (2013) [21] propose the model driven by a family of Lévy processes called $\beta$-process and exploit the Weiner-Hopf Monte Carlo method to give the CoCos price. Corcuera and Valdivia (2016) [22] propose one-sided CGMY Lévy process to model equity price dynamics and give the CoCos price by combining the closedform expression for CoCos price up to Laplace transform with numerical Fourier inversion. The present paper follows these works and proposes three first-passage time models driven by drifted Brownian motion, spectrally negative Lévy process, and double exponential jump diffusion process to price CoCos. Here the equity derivatives models are called the first-passage time models since their results are easily extended to structural models to study CoCos role and behaviours. Since pricing CoCos is our main concern and equity derivatives model has proved to be more practical, only the equity derivatives models driven by drifted Brownian motion, spectrally negative Lévy process, and double exponential jump diffusion process will be discussed. To make the results comprehensible in a more general way, these equity derivative models would be called first-passage time models in the whole paper. These models provide closed-form expressions while the latter two possess them up to Laplace transforms, whose pricing results are given by combining with numerical 
Fourier inversion and Laplace inversion. Though the model driven by drifted Brownian motion has been given by De Spiegeleer and Schoutens (2012) [11], the result given under the general Lévy framework can provide different insight and it would become more convenient to compare the BlackScholes model with the other two models under the general Lévy framework. The model driven by one-sided CGMY Lévy process is a specific and practical case of the model driven by spectrally negative Lévy process. Since the design and function of CoCos determines the financial products are very sensitive to extreme events, the models driven by spectrally negative Lévy process and double exponential jump diffusion process are natural improvement in contrast with the Black-Scholes models by incorporating jumps from these extreme events. The closed form expressions make these models able to accomplish an accurate and fast pricing of CoCos.

The present paper first develops a general Lévy framework for the first-passage time model to price CoCos with all-at-once conversion. The Lévy framework shows hybrid nature of CoCos and reduces the pricing problem to the first-passage time problem of two different Lévy processes, in which one is the measure transform result of another one where the measure transform is fixed and specific. Since rare forms of Lévy process have solved first-passage time problem, these two related Lévy processes under the Lévy framework should be in these rare forms of Lévy process so that closedform expressions for CoCos price could be provided. To avoid appearance of Lévy process whose first-passage time problem has not been solved, one simple solution is to keep this form of Lévy process unchanged under the measure transform and moreover this form of Lévy process should have solved firstpassage time problem. Taking great uncertainty about form change under the measure transform into consideration, this solution is much simpler than another solution that the form changes under the measure transform while requiring that both of these two forms of Lévy process have solved first-passage time problem. According to characteristics of new Lévy measure after the measure transform, this paper provides three specific Lévy models keeping the form of the driving Lévy process unchanged under the measure transform. Numerical results show that the models driven by spectrally negative Lévy process and double exponential jump diffusion process give more accurate CoCos price by taking jumps phenomenon in financial market into consideration.

The rest of this paper is organized as follows. Section 2 will show the general Lévy framework of the first-passage time model. Section 3 will discuss the motivation and specific expressions for these three Lévy models. Section 4 will discuss related numerical results.

\section{General Lévy Framework for CoCos Pricing}

We consider a financial institution issuing CoCos with an accounting trigger and all-at-once conversion. We follow the same assumption as in De Spiegeleer and Schoutens (2012) [11] and Corcuera et al. (2013) [21] and assume that the noncontinuously observable accounting trigger is breached when the continuous observable stock price process falls under an implied stock price barrier, which changes the trigger process from accounting index process into stock price process and transforms pricing of CoCos with an accounting trigger into pricing of CoCos with a simple market trigger. Then the trigger process becomes the stock price process of issuing firm. The trigger process is assumed to follow the exponential Lévy process under risk neutral probability measure $Q$ as

$$
S_{t}=S_{0} e^{X_{t}}, \quad t \geq 0,
$$

where $S_{0}$ is the initial value and $X_{t}$ is a general Lévy process. According to the Lévy-Itô decomposition, $X_{t}$ can be written in a general form:

$$
\begin{aligned}
X_{t}= & \mu t+\sigma W_{t}+\int_{0}^{t} \int_{|x| \geq 1} x N(\mathrm{~d} s, \mathrm{~d} x) \\
& +\int_{0}^{t} \int_{|x|<1} x(N(\mathrm{~d} s, \mathrm{~d} x)-v(\mathrm{~d} x) \mathrm{d} s),
\end{aligned}
$$

where $\mu \in \mathbb{R}, \sigma^{2} \geq 0, W$ is a standard Brownian motion, $N$ is a Poisson random measure on $([0, \infty) \times \mathbb{R}, \mathscr{B}([0, \infty)) \otimes \mathscr{B}(\mathbb{R}))$, and $v$ is a measure on $\mathbb{R} \backslash\{0\}$ with $\int_{-\infty}^{\infty}\left(1 \wedge x^{2}\right) v(\mathrm{~d} x)<\infty$. $(\mu, \sigma, v(\mathrm{~d} x))$ fully determining the Lévy process $X_{t}$ is termed as Lévy triplet and the measure $v$ is called the Lévy measure of $X_{t}$. If the Lévy process $X_{t}$ satisfies $\int_{|x|<1}|x| v(\mathrm{~d} x)<\infty$, $\int_{0}^{t} \int_{|x|<1} x N(\mathrm{~d} s, \mathrm{~d} x)<\infty$ and $\mu$ and $-\int_{|x|<1} x v(\mathrm{~d} x)$ can be combined as $d=\mu-\int_{|x|<1} x v(\mathrm{~d} x)$. In this case the Lévy process $X_{t}$ can be expressed as

$$
X_{t}=d t+\sigma W_{t}+\int_{0}^{t} \int_{\mathbb{R}} x N(\mathrm{~d} s, \mathrm{~d} x) .
$$

For more about Lévy process one can refer to Tankov (2003) [23], Kyprianou (2006) [24], and Applebaum (2009) [25].

Since $\left(e^{-r t} S_{t}\right)_{t \geq 0}$ is a martingale under the probability measure $Q$, the additional restrictions on the Lévy triplet can be given by Itô formula for semimartingales or Proposition 3.18 in Tankov (2003) [23] as

$$
\begin{aligned}
\int_{|x| \geq 1} e^{x} v(\mathrm{~d} x)< & +\infty \\
\mu= & r-\frac{1}{2} \sigma^{2} \\
& -\int_{-\infty}^{\infty}\left(e^{y}-1-y I_{|y| \leq 1}\right) v(\mathrm{~d} y) .
\end{aligned}
$$

The implied stock price barrier is assumed as $b$ where $b<$ $S_{0}$ and the accounting trigger is breached when the trigger process $S_{t}$ crosses the boundary $b$. The trigger event occurs at the first-passage time $\tau_{b}$ where $X_{t}$ crosses the boundary $\log \left(b / S_{0}\right)$ and $\tau_{b}$ can be expressed as $\tau_{b}=\inf \left\{t: X_{t} \leq\right.$ $\left.\log \left(b / S_{0}\right)\right\}$. If triggered, the CoCos per share convert into $C$ shares of equity. Denote the maturity as $T$, the principal payment at maturity as $B$, the stream of fixed coupon payments at times $0<t_{1}<\cdots<t_{m}=T$ as $c_{i}$, and the riskless interest rate as $r$. Assuming that there are no dividends after 
the conversion time $\tau_{b}$, receiving $C S_{\tau_{b}}$ at time $\tau_{b}$ is equivalent to receiving $C S_{T}$ at time $T$. Then the discounted payoffs of the components of the CoCos can be given as follows:

(i) principal payment at maturity:

$$
e^{-r T} B I_{\tau_{b}>T}
$$

(ii) coupon payment:

$$
\sum_{i=1}^{m} c_{i} B e^{-r t_{i}} I_{\tau_{b}>t_{i}}
$$

(iii) equity value through conversion:

$$
C e^{-r T} S_{T} I_{\tau_{b} \leq T}
$$

The value of CoCos can be expressed as

V

$$
\begin{aligned}
= & E_{\mathrm{Q}}\left(e^{-r T} B I_{\tau_{b}>T}+\sum_{i=1}^{m} c_{i} B e^{-r t_{i}} I_{\tau_{b}>t_{i}}+C e^{-r T} S_{T} I_{\tau_{b} \leq T}\right) \\
= & e^{-r T} B Q\left(\tau_{b}>T\right)+\sum_{i=1}^{m} c_{i} B e^{-r t_{i}} Q\left(\tau_{b}>t_{i}\right) \\
& +C e^{-r T} E_{Q}\left(S_{T} I_{\tau_{b} \leq T}\right) .
\end{aligned}
$$

Let $\psi(x, t ; \mu, \sigma, v)=Q\left(\min _{0 \leq s \leq t} X(s) \leq x\right)$. Then

$$
\begin{aligned}
V= & e^{-r T} B Q\left(\tau_{b}>T\right)+\sum_{i=1}^{m} c_{i} B e^{-r t_{i}} Q\left(\tau_{b}>t_{i}\right) \\
& +C e^{-r T} E_{\mathrm{Q}}\left(S_{T} I_{\tau_{b} \leq T}\right) \\
= & e^{-r T} B\left(1-\psi\left(\log \frac{b}{S_{0}}, T ; \mu, \sigma, v\right)\right) \\
& +\sum_{i=1}^{m} c_{i} B e^{-r t_{i}}\left(1-\psi\left(\log \frac{b}{S_{0}}, t_{i} ; \mu, \sigma, v\right)\right) \\
& +C e^{-r T} E_{\mathrm{Q}}\left(S_{T} I_{\tau_{b} \leq T}\right)=A_{1}+A_{2}+A_{3},
\end{aligned}
$$

where $A_{1}, A_{2}, A_{3}$ represent first, second, and third items, respectively.

Define new probability measure $Q^{*}$ as $\mathrm{dQ}^{*} / \mathrm{d} Q=$ $e^{-r T}\left(S_{T} / S_{0}\right)=e^{X_{T}-r T}$. According to Proposition 9.8 in Tankov (2003) [23] about the general result of equivalence of measures for Lévy processes, $X_{t}$ is a new Lévy process under probability measure $Q^{*}$. The measure transform mentioned in the whole paper refers in particular to this measure transform. Denote $X_{t}$ under $Q^{*}$ as

$$
\begin{aligned}
X_{t}= & \mu^{*} t+\sigma^{*} W_{t}^{*}+\int_{0}^{t} \int_{|x| \geq 1} x N^{*}(\mathrm{~d} s, \mathrm{~d} x) \\
& +\int_{0}^{t} \int_{|x|<1} x\left(N^{*}(\mathrm{~d} s, \mathrm{~d} x)-v^{*}(\mathrm{~d} x) \mathrm{d} s\right) .
\end{aligned}
$$

Then from Proposition 9.8 in Tankov (2003) [23] the elements of new Lévy triplet can be given as

$$
\begin{aligned}
\mu^{*} & =\mu+\sigma^{2}+\int_{-1}^{1} x\left(e^{x}-1\right) v(\mathrm{~d} x), \\
\sigma^{*} & =\sigma \\
v^{*}(\mathrm{~d} x) & =e^{x} v(\mathrm{~d} x) .
\end{aligned}
$$

If $\int_{|x|<1}|x| v^{*}(\mathrm{~d} x)<\infty$, denote $d^{*}=\mu^{*}-\int_{|x|<1} x v^{*}(\mathrm{~d} x)$ and the relation expression between $\mu^{*}$ and $\mu$ can be simplified as

$$
d^{*}=d+\sigma^{2}
$$

Then

$$
\begin{aligned}
A_{3} & =C e^{-r T} E_{Q}\left(S_{T} I_{\tau_{b} \leq T}\right) \\
& =C e^{-r T} E_{Q^{*}}\left(S_{T} I_{\tau_{b} \leq T} e^{r T} \frac{S_{0}}{S_{T}}\right)=C S_{0} Q^{*}\left(\tau_{b} \leq T\right) \\
& =C S_{0} \psi\left(\log \frac{b}{S_{0}}, T ; \mu^{*}, \sigma^{*}, v^{*}\right) .
\end{aligned}
$$

The value of CoCos can be finally given as

$$
\begin{aligned}
V= & e^{-r T} B\left(1-\psi\left(\log \frac{b}{S_{0}}, T ; \mu, \sigma, v\right)\right) \\
& +\sum_{i=1}^{m} c_{i} B e^{-r t_{i}}\left(1-\psi\left(\log \frac{b}{S_{0}}, t_{i} ; \mu, \sigma, v\right)\right) \\
& +C S_{0} \psi\left(\log \frac{b}{S_{0}}, T ; \mu^{*}, \sigma^{*}, v^{*}\right) .
\end{aligned}
$$

Equation (15) reflects hybrid nature of CoCos. If $A_{1}+A_{2}$ is termed as debt part and $A_{3}$ is termed as equity part in (10), the trigger level $b$ has a favorable effect on equity part but an adverse effect on debt part. The CoCos pricing result is the trade-off between these two effects.

Equation (15) also shows that the CoCos pricing problem is the first-passage time problem of two different Lévy processes (2) and (11). The access to giving closed-form expression for CoCos price is to make the Lévy processes (2) and (11) have closed-form expressions for their first-passage time distributions. Since rare forms of Lévy process have solved first-passage time problem, it is not easy to give closedform expression for CoCos price if these two Lévy processes have different forms. Taking the connection between these two Lévy processes into consideration, one simple solution is to keep this form of Lévy process unchanged under the measure transform while requiring that this from of Lévy process should have solved the first-passage time problem. According to the characteristics of new Lévy measure after the measure transform, we will propose three Lévy models whose driving Lévy processes keep the form unchanged under the measure transform and have closed-form expression for the firstpassage time distribution while two of them possess closedform expressions up to Laplace transform. The specific results will be discussed in Section 3. 
Remark 1. The model covers the pricing of write-down CoCos. If conversion into $C$ shares of equity is replaced by payment of $C$ units of currency, it is convenient to obtain the CoCos price by replacing $C e^{-r T} S_{T} I_{\tau_{b} \leq T}$ by $C e^{-r T} I_{\tau_{b} \leq T}$ without considering the measure transform. If the CoCos are assumed to have a continuous coupon rate of $c$ as in Glasserman and Nouri (2012) [10] for simple treatment, the coupon payment will turn into $\int_{0}^{T} c B e^{-r t} I_{\tau_{b}>t} \mathrm{~d} t$. Then $E_{\mathrm{Q}}\left(\int_{0}^{T} c B e^{-r t} I_{\tau_{b}>t} \mathrm{~d} t\right)=$ $\int_{0}^{T} c B e^{-r t} \mathrm{Q}\left(\tau_{b}>t\right) \mathrm{d} t$. The easy evaluation of $c B e^{-r t} \mathrm{Q}\left(\tau_{b}>t\right)$ makes the time integral $\int_{0}^{T} c B e^{-r t} \mathrm{Q}\left(\tau_{b}>t\right) \mathrm{d} t$ can be easily and accurately evaluated by numerical integration formulas, such as Newton-Cotes formula.

Remark 2. The price of CoCos whose trigger process is described by exponential Lévy process can be evaluated through some other numerical techniques such as Monte Carlo simulation and solving integrodifferential equations. However, the Monte Carlo simulation is biased and slow. When the Monte Carlo simulation is applied to estimate the distribution of first-passage time of Lévy process, the systematic discretization bias resulting from approximating a continuous time process by a discrete time process in simulation is serious due to the presence of the boundary and jumps, both theoretically and numerically. Kou and Wang (2003) [26] have discussed the situation about double exponential jump diffusion process. Comparing to get the solution from solving integrodifferential equations, the probabilistic approach given in this paper can provide more insights into the CoCos pricing problem.

\section{Discussion and Result}

To keep the form of Lévy process unchanged under the measure transform, only the new Lévy measure $v^{*}$ in Lévy triplet $\left(\mu^{*}, \sigma^{*}, v^{*}\right)$ needs be considered. Observing the Lévy measure $v^{*}(\mathrm{~d} x)=e^{x} v(\mathrm{~d} x)$, the simplest case to make (2) and (11) have the same form is $v=0$. In this case, both (2) and (11) become drifted Brownian motion. Since drifted Brownian motion has closed-form expression for first-passage time distribution, the closed-form expression for CoCos price can be provided through (15). Based on sample space of Lévy measure unchanged under the measure transform, a large family of Lévy processes called spectrally one-side Lévy process can come to mind. Assuming that $v \neq 0$ and $\sigma \neq 0$, spectrally one-side Lévy process can be defined as $v(0, \infty)=$ 0 or $v(-\infty, 0)=0$. After the measure transform, $v^{*} \neq 0$, $\sigma^{*} \neq 0$ and $v^{*}$ still satisfies $v^{*}(0, \infty)=0$ or $v^{*}(-\infty, 0)=0$. In this case, both (2) and (11) become spectrally one-side Lévy process. The closed-form expression for Laplace transform of the first-passage time distribution for spectrally oneside Lévy process has been obtained by Rogers (2000) [27]. Then the CoCos price can be given by combining the result with Laplace inverse algorithm and (15). Since downward risk of trigger process is more worthy to be considered in CoCos pricing, only the spectrally negative Lévy process will be discussed in this case. Since $v(\mathbb{R})<\infty$ can lead to $v^{*}(\mathbb{R})<$ $\infty$, the Lévy process in jump diffusion form with two-sided jumps is considered and the jump diffusion form keeps unchanged under the measure transform. Since not all the jump diffusion processes solve the first-passage time problems, the jump diffusion form should be more specific and the scope of this form should be smaller. Since specification of jump form could reduce the scope of jump diffusion form and the only difference between Lévy measure $v$ and $v^{*}$ is a multiplier in the exponential form, jump distribution of the Lévy process in jump diffusion form should be limited to the family of exponential type distribution to possibly make (2) and (11) have the same form when the form becomes more specific. A simple case is the double exponential jump diffusion process whose first-passage time problem has been solved by Kou and Wang (2003) [26] by means of the conditional memoryless of exponential distribution. The property keeping the double exponential jump diffusion form unchanged under the measure transform will be proved below. Then the corresponding CoCos price can also be given through (15). The detailed results of these three cases will be discussed below.

3.1. Drifted Brownian Motion. Let $v=0$; then $X_{t}=\mu t+\sigma W_{t}$, whose first-passage time distribution is given as

$$
P\left(\gamma_{x} \leq t\right)=\phi\left(\frac{x-\mu t}{\sigma \sqrt{t}}\right)+e^{2 x \mu / \sigma^{2}} \phi\left(\frac{x+\mu t}{\sigma \sqrt{t}}\right)
$$

where $\phi$ is the cumulative function of standard normal distribution and $\gamma_{x}=\inf \left\{t: X_{t} \leq x\right\}$. Since $v=0$ leads to $v^{*}=$ 0 , (11) is still a drifted Brownian motion. The CoCos price has closed-form expression summarized as in Proposition 3.

Proposition 3. The first-passage time model driven by drifted Brownian motion gives CoCos price as

$$
\begin{aligned}
V= & e^{-r T} B\left(1-\psi\left(\log \frac{b}{S_{0}}, T ; \mu, \sigma, v\right)\right) \\
& +\sum_{i=1}^{m} c_{i} B e^{-r t_{i}}\left(1-\psi\left(\log \frac{b}{S_{0}}, t_{i} ; \mu, \sigma, v\right)\right) \\
& +C S_{0} \psi\left(\log \frac{b}{S_{0}}, T ; \mu^{*}, \sigma^{*}, v^{*}\right),
\end{aligned}
$$

where $b$ is a prespecified trigger level, $\mu^{*}=\mu+\sigma^{2}, \sigma^{*}=\sigma$, and $\psi(x, t ; \mu, \sigma, v)=\phi((x-\mu t) / \sigma \sqrt{t})+e^{2 x \mu / \sigma^{2}} \phi((x+\mu t) / \sigma \sqrt{t})$ where $\phi$ is the cumulative function of standard normal distribution.

Remark 4. Though this result has been given by De Spiegeleer and Schoutens (2012) [11], this result given under the Lévy framework can provide different insight and more importantly the expression in Proposition 3 makes the models comparison under the Lévy framework become more intuitive and convenient.

3.2. Spectrally Negative Lévy Process. Let $v \neq 0, \sigma \neq 0$, and $v(0, \infty)=0$; then $X_{t}$ becomes spectrally negative Lévy process. Since these conditions lead to $v^{*} \neq 0, \sigma^{*} \neq 0$, and $v^{*}(0, \infty)=0,(11)$ is still a spectrally negative Lévy process. 
The closed-form expression for Laplace transform of firstpassage time distribution of spectrally negative Lévy process has been given by Rogers (2000) [27] through Wiener-Hopf factorisation. Rogers (2000) [27] gives the first-passage time distribution through approximating the standard Fourier inversion transform, which exploits suitable integration contour change to sidestep the difficulty in directly solving the equation of Laplace exponent. The related result is given below. For Details and proofs of the following result one can refer to Rogers (2000) [27].

Let $\gamma_{x}=\inf \left\{t: X_{t} \leq x\right\}, f(t, x)=P\left(\gamma_{-x}>t\right)$, $\bar{X}_{t}=\sup _{s \leq t} X_{s}$, and let $e_{\theta}$ be an exponential distribution with parameter $\theta$. Based on the exponential law of $\bar{X}_{e_{\theta}}$ and WienerHopf factorisation, the Laplace transform of $f(t, x)$ can be expressed as

$$
\begin{aligned}
\tilde{f}(\theta, z) & =\int_{0}^{\infty} \int_{0}^{\infty} e^{-\theta t-z x} f(t, x) \mathrm{d} t \mathrm{~d} x \\
& =\frac{\beta^{*}(\theta)-z}{(\theta-\varphi(z)) \beta^{*}(\theta) z},
\end{aligned}
$$

where $\varphi(z)$ is Laplace exponent of spectrally negative Lévy process characterized by $E\left(e^{z X_{t}}\right)=e^{t \varphi(z)}$ and represented as

$$
\begin{aligned}
\varphi(z)= & \mu z+\frac{1}{2} \sigma^{2} z^{2} \\
& +\int_{-\infty}^{0}\left\{e^{z x}-1-z x I_{|x| \leq 1}\right\} v(\mathrm{~d} x),
\end{aligned}
$$

$\beta^{*}(\theta)$ a solution to $\varphi(\beta)=\theta$. Since $\sigma \neq 0$, the difficulty in directly evaluating $\beta^{*}(\theta)$ when the numerical Fourier inversion is implemented to obtain $f(t, x)$ can be overcome by replacing $\beta^{*}(\theta)$ by $\varphi_{0}{ }^{-1}(\theta)$ where $\varphi_{0}(z)=\mu z+(1 / 2) \sigma^{2} z^{2}$ and $\varphi_{0}(z)$ approximates $\varphi(z)$ and has available closed-form inverse function. This replacement can be through the substitution of suitable integration contour in Fourier inversion. The algorithm to evaluate the first-passage time distribution of spectrally negative Lévy process can be summarized as the following lemma.

Lemma 5. For fixed $t$ and $x$ and given the parameter set $\left(A_{1}, A_{2}, l_{1}, l_{2}, N\right)$, define $\gamma_{x}=\inf \left\{t: X_{t} \leq x\right\} . P\left(\gamma_{x} \leq t\right)$ can be given as

$$
P\left(\gamma_{x} \leq t\right)=1-f(t,-x)
$$

where

$$
f(t, x) \approx \sum_{k=0}^{M} 2^{-M}\left(\begin{array}{c}
M \\
k
\end{array}\right) S_{N+k}
$$

where

$$
\begin{aligned}
S_{N} & =\frac{h_{1} h_{2}}{4 \pi^{2}} \sum_{n=-N}^{N} \sum_{m=-N}^{N} g^{\prime}\left(a_{1}+i n h_{1}\right) \\
& \cdot \tilde{f}\left(g\left(a_{1}+i n h_{1}\right), a_{2}+i m h_{2}\right) \\
& \times \exp \left\{\operatorname{tg}\left(a_{1}+i n h_{1}\right)+x\left(a_{2}+i m h_{2}\right)\right\},
\end{aligned}
$$

$a_{1}=A_{1} /\left(2 t l_{1}\right), a_{2}=A_{2} /\left(2 x l_{2}\right), h_{1}=\pi /\left(t l_{1}\right), h_{2}=\pi /\left(x l_{2}\right)$, $g=\varphi \circ \varphi_{0}^{-1}$, and

$$
\tilde{f}(g(\varsigma), z)=\frac{\varphi_{0}^{-1}(\varsigma)-z}{(g(\varsigma)-\varphi(z)) \varphi_{0}^{-1}(\varsigma) z},
$$

where $\varphi(z)=\mu z+(1 / 2) \sigma^{2} z^{2}+\int_{-\infty}^{0}\left\{e^{z x}-1-z x I_{|x| \leq 1}\right\} v(\mathrm{~d} x)$, $\varphi_{0}^{-1}(z)=\left(\sqrt{\mu^{2}+2 \sigma^{2} z}-\mu\right) / \sigma^{2}$.

Here $l_{1}$ and $l_{2}$ are positive integers, and $A_{1}$ and $A_{2}$ are positive reals chosen large enough to control the aliasing error. They are suggested to take $A_{1}=A_{2}=22$ and $l_{1}=l_{2}=1$ to give satisfactory results. For further details one can refer to Rogers (2000) [27] and the references therein. Given the expression to evaluate the first-passage time distribution of spectrally negative Lévy process, the expression to evaluate CoCos price can be given through (15) as in Proposition 6.

Proposition 6. The first-passage time model driven by spectrally negative Lévy Process gives CoCos price as

$$
\begin{aligned}
V= & e^{-r T} B\left(1-\psi\left(\log \frac{b}{S_{0}}, T ; \mu, \sigma, v\right)\right) \\
& +\sum_{i=1}^{m} c_{i} B e^{-r t_{i}}\left(1-\psi\left(\log \frac{b}{S_{0}}, t_{i} ; \mu, \sigma, v\right)\right) \\
& +C S_{0} \psi\left(\log \frac{b}{S_{0}}, T ; \mu^{*}, \sigma^{*}, v^{*}\right),
\end{aligned}
$$

where $\psi(x, t ; \mu, \sigma, v)=1-f(t,-x)$ and $b$ is a prespecified trigger level:

$$
\begin{aligned}
\mu^{*} & =\mu+\sigma^{2}+\int_{-1}^{0} x\left(e^{x}-1\right) v(\mathrm{~d} x), \\
\sigma^{*} & =\sigma, \\
v^{*}(\mathrm{~d} x) & =e^{x} v(\mathrm{~d} x), \\
f(t, x) & \approx \sum_{k=0}^{M} 2^{-M}\left(\begin{array}{c}
M \\
k
\end{array}\right) S_{N+k}
\end{aligned}
$$

where $S_{N}$ is the same notation as in Lemma 5.

Spectrally negative Lévy process is a large family of Lévy processes including various jump forms. To evaluate CoCos price through Proposition 6, the jump form of spectrally negative Lévy process need not keep unchanged under the measure transform. Two simple examples are given below. The jump form in Example 7 does not change under the measure transform while the jump form in Example 8 changes. Both of them can evaluate CoCos price through Proposition 6.

Example 7. This example uses jumps that have an exponential distribution with parameter $\eta$, arriving at rate $\lambda$. The Laplace exponent can be expressed as

$$
\varphi(z)=\left(\mu+\lambda\left(\frac{1-e^{-\eta}}{\eta}-e^{-\eta}\right)\right) z+\frac{1}{2} \sigma^{2} z^{2}-\frac{\lambda z}{\eta+z}
$$


After the measure transform, the jumps have an exponential distribution with density function $F^{*}(\mathrm{~d} x)=(\eta+1) e^{(\eta+1) x}$, arriving at rate $\lambda^{*}=\lambda \eta /(\eta+1)$ under the probability measure $Q^{*}$. The jump distribution is still an exponential distribution after the measure transform. The Laplace exponent can be expressed as

$$
\begin{aligned}
\varphi^{*}(z)= & \left(\mu+\sigma^{2}+\lambda\left(\frac{1-e^{-\eta}}{\eta}-e^{-\eta}\right)\right) z+\frac{1}{2} \sigma^{2} z^{2} \\
& -\frac{\lambda \eta z}{(\eta+1)(\eta+z+1)} .
\end{aligned}
$$

The CoCos price can be evaluated through Proposition 6.

Example 8. The jumps of spectrally negative Lévy process are assumed to be uniformly distributed on the interval $[-c, 0]$, where $c \geq 1$, arriving at rate $\lambda$. The Laplace exponent can be expressed as

$$
\varphi(z)=\left(\mu+\frac{\lambda}{2 c}\right) z+\frac{1}{2} \sigma^{2} z^{2}+\frac{\lambda\left(1-e^{-c z}-c z\right)}{c z} .
$$

After the measure transform, the jump distribution has density function $F^{*}(\mathrm{~d} x)=e^{x} /\left(1-e^{-c}\right)$ defined on the interval $[-c, 0]$, arriving at rate $\lambda^{*}=\lambda\left(1-e^{-c}\right) / c$ under the probability measure $Q^{*}$. The Laplace exponent can be expressed as

$$
\begin{aligned}
\varphi^{*}(z)= & \left(\mu+\sigma^{2}+\frac{\lambda}{2 c}\right) z+\frac{1}{2} \sigma^{2} z^{2} \\
& +\frac{\lambda}{c}\left[\frac{1-e^{-c(z+1)}}{z+1}+e^{-c}-1\right] .
\end{aligned}
$$

The form of jump distribution has changed under the measure transform, but the CoCos price still can be evaluated through Proposition 6 since both (2) and (11) are still spectrally negative Lévy processes in this case.

Remark 9. The one-sided CGMY Lévy model proposed by Corcuera and Valdivia (2016) [22] is a practical and specific case of Proposition 6. Since downward risk always needs to be considered in financial market, spectrally negative Lévy process has been widely applied in finance. From this point Proposition 6 enriches the finance applications of spectrally negative Lévy process.

3.3. Double Exponential Jump Diffusion Process. Double exponential jump diffusion process is a Lévy process of jump diffusion form

$$
X_{t}=d t+\sigma W_{t}+\sum_{i=1}^{N(t)} Y_{i}
$$

where $\left\{W_{t} ; t \geq 0\right\}, d$, and $\sigma$ are the same notations as in (3), $N_{t}$ is a Poisson process with rate $\lambda$, and the jump sizes $\left\{Y_{1}, Y_{2}, \ldots\right\}$ independent and identically distributed random variables have an asymmetric double exponential distribution with the density function:

$$
f_{Y}(y)=p \eta_{1} e^{-\eta_{1} y} I_{\{y \geq 0\}}+q \eta_{2} e^{\eta_{2} y} I_{\{y<0\}}
$$

where $\eta_{1}>1, \eta_{2}>0, p \geq 0, q \geq 0$, and $p+q=$ 1. The random processes $\left\{W_{t} ; t \geq 0\right\},\left\{N_{t} ; t \geq 0\right\}$ and random variables $\left\{Y_{1}, Y_{2}, \ldots\right\}$ are independent. The double exponential jump diffusion process is a special Lévy process whose Lévy measure $v$ satisfies $v(\mathbb{R})<\infty$. The Laplace exponent can be given as

$$
\psi(z)=d z+\frac{1}{2} \sigma^{2} z^{2}+\lambda\left(\frac{p \eta_{1}}{\eta_{1}-z}+\frac{q \eta_{2}}{\eta_{2}+z}-1\right) .
$$

Lévy measure of double exponential jump diffusion process can be expressed as

$$
v(d y)=\lambda f_{Y}(y) d y .
$$

Since $v(\mathbb{R})<\infty$ leads to $v^{*}(\mathbb{R})<\infty$, the double exponential jump diffusion process (31) under the probability measure $Q^{*}$ is a new Lévy process in jump diffusion form

$$
X_{t}=d^{*} t+\sigma^{*} W_{t}^{*}+\sum_{i=1}^{N^{*}(t)} Y_{i}^{*}
$$

where $d^{*}$ is the same notation as in (13). From (12) and (13) $d^{*}$ and $\sigma^{*}$ here still satisfy $d^{*}=d+\sigma^{2}, \sigma^{*}=\sigma$. Denoting $\omega=E_{Q}\left(e^{Y}\right)=p \eta_{1} /\left(\eta_{1}-1\right)+q \eta_{2} /\left(\eta_{2}+1\right)$, the new Poisson process $N^{*}(t)$ under the new probability measure $Q^{*}$ has a new rate $\lambda^{*}=\lambda E_{\mathrm{Q}}\left(e^{Y}\right)=\lambda \omega$ and the density of the new jump size $Y^{*}$ is given by

$$
\begin{aligned}
& f_{Y^{*}}(y)=\frac{e^{y} f_{Y}(y)}{E_{\mathrm{Q}}\left(e^{Y}\right)}=\frac{1}{\omega}\left(p \eta_{1} e^{-\left(\eta_{1}-1\right) y} I_{\{y \geq 0\}}\right. \\
& \left.+q \eta_{2} e^{\left(\eta_{2}+1\right) y} I_{\{y<0\}}\right) \\
& =\frac{1}{\omega}\left(\frac{p \eta_{1}}{\eta_{1}-1}\left(\eta_{1}-1\right) e^{-\left(\eta_{1}-1\right) y} I_{\{y \geq 0\}}\right. \\
& \left.+\frac{q \eta_{2}}{\eta_{2}+1}\left(\eta_{2}+1\right) e^{\left(\eta_{2}+1\right) y} I_{\{y<0\}}\right) .
\end{aligned}
$$

The jump distribution under the probability measure $Q^{*}$ is still a double exponential distribution with parameters $\eta_{1}^{*}=$ $\eta_{1}-1, \eta_{2}^{*}=\eta_{2}+1, p^{*}=(1 / \omega)\left(p \eta_{1} /\left(\eta_{1}-1\right)\right)$, and $q^{*}=$ $(1 / \omega)\left(q \eta_{2} /\left(\eta_{2}+1\right)\right)$, which proves that the double exponential jump diffusion process under the measure transform is still a double exponential jump diffusion process; that is, both (31) and (35) are double exponential jump diffusion processes. The Laplace transform of the first-passage time distribution of double exponential jump diffusion process has been obtained by Kou and Wang (2003) [26]. They exploit numerical Laplace inversion called Gaver-Stehfest algorithm to evaluate the first-passage time distribution. The related result is given below. For details and proofs of the related results one can refer to Kou and Wang (2003) [26].

Denote $f(t)=P\left(\gamma_{x} \leq t\right)$ where $\gamma_{x}$ is still defined as $\gamma_{x}=$ $\inf \left\{t: X_{t} \leq x\right\}$. The Laplace transform of $f(t)$ can be given through integration by part as

$$
\begin{aligned}
\widehat{f}(\alpha) & =\int_{0}^{\infty} e^{-\alpha t} P\left(\gamma_{x} \leq t\right) \mathrm{d} t \\
& =\frac{1}{\alpha} \int_{0}^{\infty} e^{-\alpha t} \mathrm{~d} P\left(\gamma_{x} \leq t\right)=\frac{1}{\alpha} E\left(e^{-\alpha \gamma_{x}}\right) .
\end{aligned}
$$


If given $E\left(e^{-\alpha \gamma_{x}}\right)$, the Laplace transform of $f(t)$ can be obtained through (37). $E\left(e^{-\alpha \gamma_{x}}\right)$ will be given below through Theorem 3.1 in Kou and Wang (2003) [26].

Denote $\beta_{1, \alpha}, \beta_{2, \alpha},-\beta_{3, \alpha},-\beta_{4, \alpha}$ as four roots of the Laplace exponent equation $\psi(z)=\alpha$ satisfying

$$
\begin{aligned}
& 0<\beta_{1, \alpha}<\eta_{1}<\beta_{2, \alpha}<\infty, \\
& 0<\beta_{3, \alpha}<\eta_{2}<\beta_{4, \alpha}<\infty .
\end{aligned}
$$

Denoting $\xi_{x}=\inf \left\{t: X_{t} \geq x\right\}$, Theorem 3.1 in Kou and Wang (2003) [26] gives the Laplace transform of $\xi_{x}$ as

$$
\begin{aligned}
E\left(e^{-\alpha \xi_{x}}\right)= & \frac{\eta_{1}-\beta_{1, \alpha}}{\eta_{1}} \frac{\beta_{2, \alpha}}{\beta_{2, \alpha}-\beta_{1, \alpha}} e^{-x \beta_{1, \alpha}} \\
& +\frac{\beta_{2, \alpha}-\eta_{1}}{\eta_{1}} \frac{\beta_{1, \alpha}}{\beta_{2, \alpha}-\beta_{1, \alpha}} e^{-x \beta_{2, \alpha}}
\end{aligned}
$$

Denote $\widetilde{X}_{t}=-X_{t}$. The parameters in $\widetilde{X}_{t}$ satisfy

$$
\begin{aligned}
\tilde{\mu} & =-\mu, \\
\widetilde{p} & =q, \\
\tilde{q} & =p, \\
\tilde{\eta}_{1} & =\eta_{2}, \\
\tilde{\eta}_{2} & =\eta_{1} .
\end{aligned}
$$

Denote Laplace exponent of $\widetilde{X}(t)$ as $\widetilde{\psi}(x)$. According to the equation $\widetilde{\psi}(x)=\psi(-x)$, the roots between $\widetilde{\psi}(x)=\alpha$ and $\psi(x)=\alpha$ are only different in sign. Since $\gamma_{x}=\inf \left\{t: \widetilde{X}_{t} \geq\right.$ $-x$, through substituting the parameters in (39) as $x \rightarrow-x$, $\eta_{1} \rightarrow \eta_{2}, \eta_{2} \rightarrow \eta_{1}, \beta_{1, \alpha} \rightarrow \beta_{3, \alpha}$, and $\beta_{2, \alpha} \rightarrow \beta_{4, \alpha}$, the Laplace transform of $\gamma_{x}$ is given as

$$
\begin{aligned}
E\left(e^{-\alpha \gamma_{x}}\right)= & \frac{\eta_{2}-\beta_{3, \alpha}}{\eta_{2}} \frac{\beta_{4, \alpha}}{\beta_{4, \alpha}-\beta_{3, \alpha}} e^{x \beta_{3, \alpha}} \\
& +\frac{\beta_{4, \alpha}-\eta_{2}}{\eta_{2}} \frac{\beta_{3, \alpha}}{\beta_{4, \alpha}-\beta_{3, \alpha}} e^{x \beta_{4, \alpha}} .
\end{aligned}
$$

Combining (37) with (41), the Laplace transform of $f(t)$ is given. Kou and Wang (2003) [26] exploit the numerical Laplace inversion called Gaver-Stehfest algorithm to evaluate the first-passage time distribution with Richardson extrapolation technique to speed up the convergence. The Laplace transform of $f(t)$ and Gaver-Stehfest algorithm are combined and the evaluation of $f(t)$ can be summarized as the following Lemma.

Lemma 10. For fixed $t$ and $x$ and given the parameter set $(n, B)$, defining $\gamma_{x}=\inf \left\{t: X_{t} \leq x\right\} . P\left(\gamma_{x} \leq t\right)$ can be given as

$$
P\left(\gamma_{x} \leq t\right) \approx \sum_{k=1}^{n} w(k, n) \widetilde{f}_{k+B}(t, x)
$$

where

$$
\begin{aligned}
w(k, n) & =(-1)^{n-k} \frac{k^{n}}{k !(n-k) !}, \\
\tilde{f}_{m}(t, x) & =\frac{\ln (2)}{t} \frac{(2 m) !}{m !(m-1) !} \sum_{k=0}^{m}(-1)^{k} \\
\cdot\left(\begin{array}{c}
m \\
k
\end{array}\right) & \hat{f}\left((m+k) \frac{\ln (2)}{t}, x\right),
\end{aligned}
$$

where

$$
\begin{gathered}
\widehat{f}(\alpha, x)=\frac{1}{\alpha}\left(\frac{\eta_{2}-\beta_{3, \alpha}}{\eta_{2}} \frac{\beta_{4, \alpha}}{\beta_{4, \alpha}-\beta_{3, \alpha}} e^{x \beta_{3, \alpha}}\right. \\
\left.+\frac{\beta_{4, \alpha}-\eta_{2}}{\eta_{2}} \frac{\beta_{3, \alpha}}{\beta_{4, \alpha}-\beta_{3, \alpha}} e^{x \beta_{4, \alpha}}\right),
\end{gathered}
$$

where $-\beta_{3, \alpha},-\beta_{4, \alpha}$ are the only two negative roots of the equation $\psi(z)=\alpha$ satisfying

$$
0<\beta_{3, \alpha}<\eta_{2}<\beta_{4, \alpha}<\infty,
$$

where $\psi(z)$ is the Laplace exponent of $X_{t}$.

Here $B \geq 0$ is the initial burning out number and typically equals 2 or 3 . The algorithm typically converges nicely even for $n$ between 5 and 10. Given the expression to evaluate the first-passage time distribution of double exponential jump diffusion process, the expression for CoCos price can be given through (15) as in Proposition 11.

Proposition 11. The first-passage time model driven by double exponential jump diffusion process gives CoCos price as

$$
\begin{aligned}
V= & e^{-r T} B\left(1-\psi\left(\log \frac{b}{S_{0}}, T ; \mu, \sigma, v\right)\right) \\
& +\sum_{i=1}^{m} c_{i} B e^{-r t_{i}}\left(1-\psi\left(\log \frac{b}{S_{0}}, t_{i} ; \mu, \sigma, v\right)\right) \\
& +C S_{0} \psi\left(\log \frac{b}{S_{0}}, T ; \mu^{*}, \sigma^{*}, v^{*}\right),
\end{aligned}
$$

where $\psi(x, t ; \mu, \sigma, v)=f(t, x)$ and $b$ is a prespecified trigger level:

$$
\begin{aligned}
\mu^{*} & =\mu+\sigma^{2}+\lambda p \eta_{1}\left(\frac{1-\eta_{1} e^{1-\eta_{1}}}{\left(1-\eta_{1}\right)^{2}}\right. \\
& \left.+\frac{\left(\eta_{1}+1\right) e^{-\eta_{1}}-1}{\eta_{1}^{2}}\right) \\
& +\lambda q \eta_{2}\left(\frac{\left(2+\eta_{2}\right) e^{-\left(1+\eta_{2}\right)}-1}{\left(1+\eta_{2}\right)^{2}}\right. \\
& \left.+\frac{1-\left(\eta_{2}+1\right) e^{-\eta_{2}}}{\eta_{2}{ }^{2}}\right) .
\end{aligned}
$$


$\sigma^{*}=\sigma, v^{*}(\mathrm{~d} x)=e^{x} v(\mathrm{~d} x)$, where

$$
f(t, x) \approx \sum_{k=1}^{n} w(k, n) \widetilde{f}_{k+B}(t, x),
$$

where $w(k, n)$ and $\widetilde{f}_{m}(t, x)$ are the same notations as in Lemma 10.

Remark 12. If $\psi$ is denoted as $\psi\left(t, x ; d, \sigma, \lambda, p, \eta_{1}, \eta_{2}\right)$, it is more concise to express the parameters' change in $\psi$ under the measure transform as

$$
\begin{aligned}
& d^{*}=d+\sigma^{2}, \\
& \sigma^{*}=\sigma, \\
& \lambda^{*}=\lambda \omega, \\
& p^{*}=\frac{1}{\omega} \frac{p \eta_{1}}{\eta_{1}-1}, \\
& q^{*}=\frac{1}{\omega} \frac{q \eta_{2}}{\eta_{2}+1}, \\
& \eta_{1}^{*}=\eta_{1}-1, \\
& \eta_{2}^{*}=\eta_{2}+1
\end{aligned}
$$

and the evaluation for CoCos price becomes more direct and convenient. However, $\psi$ expressed by the Lévy triplet in Proposition 11 can make models' comparison under the Lévy framework become more simple and intuitive.

Remark 13. Though double exponential jump diffusion process possesses two-sided jumps, it cannot claim that the model driven by double exponential jump diffusion process is more superior to the model driven by spectrally negative Lévy process. The jumps of the driving process in the former are finite activity and the corresponding jump distribution is limited to the double exponential form while that in the latter can be of infinite activity and the jump form can be various. These two models take their own advantages in CoCos pricing. However, without doubt, the model driven by double exponential jump diffusion process will perform better than the model driven by spectrally negative Lévy process only with exponential jumps. This fact is used in numerical experiment.

\section{Numerical Experiment}

We exploit numerical experiment to compare these three Lévy models. Numerical experiment investigates the difference in CoCos price among the Lévy models and how the CoCos price changes with the parameters in the models. For simple statement, the models driven by drifted Brownian motion, spectrally negative Lévy process, and double exponential jump diffusion process are abbreviated as BS, SNP, and Kou, respectively. To make comparison results more explainable, jumps of spectrally negative Lévy process are chosen to arrive at a limited rate and have an exponential distribution whose parameters are set as the same as that in negative jumps of double exponential jump diffusion process. The parameters are chosen to reflect those in typical finance application and primarily refer to Rogers (2000) [27] and Kou and Wang (2003) [26]. They are listed in Table 1. Since these Lévy models all satisfy $\int_{|x|<1}|x| v(\mathrm{~d} x)<\infty$ under the assumptions, $X_{t}$ can be expressed as (3) in the Lévy models. $\mu$ is chosen to satisfy martingale condition as in (5) and the corresponding $d$ is listed in Table 1. $\sigma$ is chosen to keep second-order moment of $X_{t}$ equal all the time in the Lévy models and the relation expressions for $\sigma$ parameters are also listed in Table 1. The parameters listed in Table 1 make the first and second order of the $X_{t}$ equal in these Lévy models all the time and these parameters can be treated as an estimation result from generalized method of moments. The parameter sets provide an intuitive explanation that BS compresses all the jumps information into volatility and SNP compresses positive jumps information into volatility. The riskless rate $r$ is assumed as 0.03 . The CoCos are assumed with a maturity of 10 years and fixed coupon $c$ of 0.08 paid every half year. Let the principal $B$ of the CoCos be 100 , the initial stock price $S_{0}$ 10 , and the implied stock price barrier $b$ 8.5. Once the trigger event occurs, the CoCos per share convert into 20 shares of equity (i.e., $C=20$ ). The parameters in numerical Fourier inverse for SNP are chosen as $l_{1}=l_{2}=1, A_{1}=A_{2}=22$, $N=6$, and $M=9$. The parameters in numerical Laplace inverse for Kou are chosen as $n=8, B=2$. Table 2 lists the parameters change under the measure transform in the Lévy models.

Figure 1(a) shows CoCos price at different $b$ values when $\lambda=0.01$. Since $\lambda$ is small enough, jumps in SNP and Kou are so few that the price evaluated from them becomes almost the same as that from BS. The graphs are nonmonotonic, which reflects hybrid nature of the CoCos. The variation is the tradeoff between the opposite effects from debt part and equity part. The CoCos price decreases when $b$ increases from 0 to 4.8 , which illustrates that the debt part dominates CoCos pricing in $(0,4.8)$. The same is true for dominant role of equity part in $(4.8,10)$.

Figure 1(b) reproduces Figure 1(a) but now with $\lambda=30$. The SNP curve and the Kou curve almost overlap. This result shows that positive jumps have limited influence on CoCos pricing since the only difference between these two models is the positive jumps information that is compressed into volatility in SNP. In contrast with Figure 1(a), the number of jumps increases largely in SNP and Kou. If 0.5 is taken as the criterion of significant difference, the CoCos price in SNP and Kou is significantly different from that in BS when $b \in(5.9,10)$. Equity part dominates the CoCos price in this region and its value becomes more and more sensitive to $b$ when $b$ increases, which makes the difference in CoCos price between BS and the other two models become bigger and bigger for their different accuracies. SNP and Kou are natural improvement in contrast with the BS since they incorporate jumps structure into BS for the characterization of jumps phenomenon in financial market. The difference between BS and the other two models shows the value and significance of introducing the SNP and Kou models. SNP and Kou have lower CoCos price than BS, which illustrates that BS would 
TABLE 1: The parameters for the three Lévy models.

\begin{tabular}{lccc}
\hline Parameter & BS & SNP & Kou \\
\hline$d$ & $r-\frac{1}{2} \sigma_{\mathrm{BS}}^{2}$ & $r-\frac{1}{2} \sigma_{\mathrm{SNP}}^{2}+\frac{\lambda_{\mathrm{SNP}}}{\eta_{2}+1}$ & $r-\frac{1}{2} \sigma_{\text {Kou }}^{2}+\frac{\lambda_{\text {Kou }} p}{1-\eta_{1}}+\frac{\lambda_{\text {Kou }}(1-p)}{\eta_{2}+1}$ \\
$\sigma$ & $\sqrt{\sigma_{\text {Kou }}^{2}+2 \lambda_{\text {Kou }}\left(\frac{p}{\eta_{1}^{2}}+\frac{1-p}{\eta_{2}^{2}}\right)}$ & $\sqrt{\sigma_{\text {Kou }}^{2}+\frac{2 \lambda_{\mathrm{Kou}} p}{\eta_{1}^{2}}}$ & 0.2 \\
$\lambda$ & $\lambda_{\text {Kou }}(1-p)$ & 8 \\
$\eta_{1}$ & $1 / 0.03$ & $1 / 0.02$ \\
$\eta_{2}$ & & $1 / 0.03$ \\
\hline
\end{tabular}

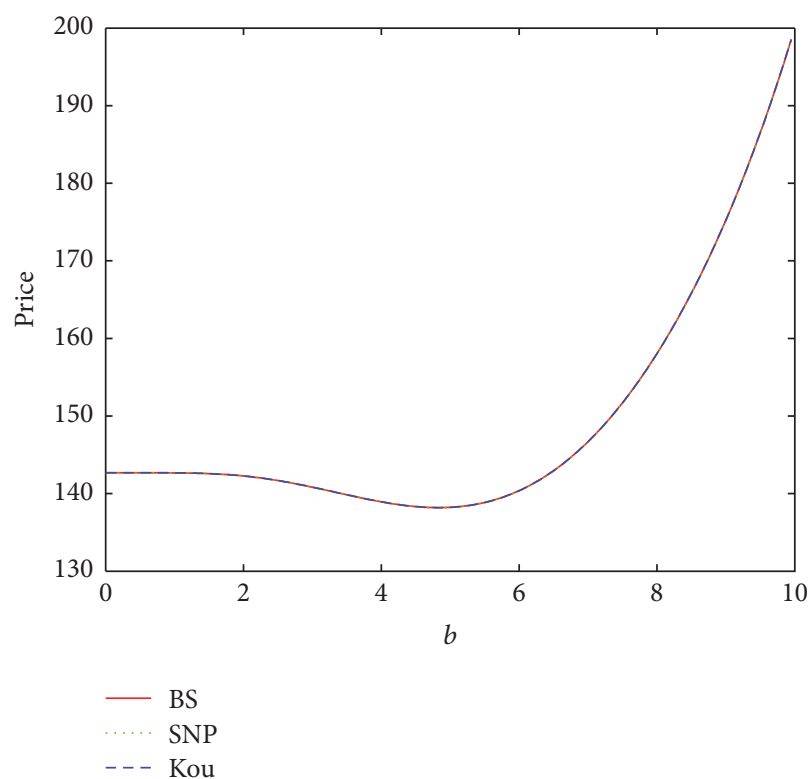

(a)

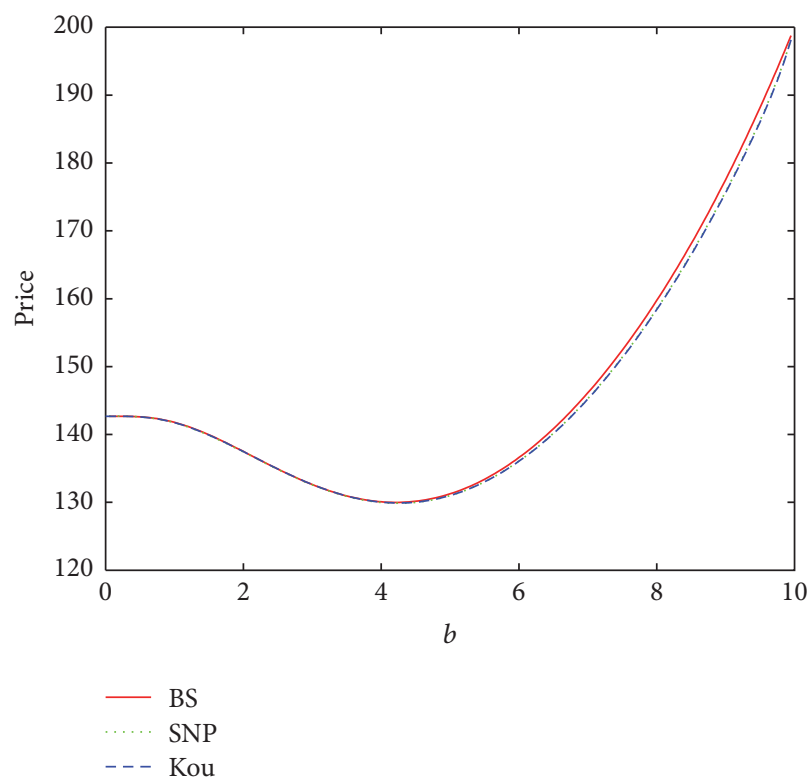

(b)

Figure 1: (a) The sensitivity of CoCos price to trigger barrier level $b$ when $\lambda=0.01$. (b) The sensitivity of CoCos price to trigger barrier level $b$ when $\lambda=30$.

TABLE 2: The parameters' change under the measure transform.

\begin{tabular}{lccc}
\hline Parameter & BS & SNP & Kou \\
\hline$d^{*}$ & $d+\sigma^{2}$ & $d+\sigma^{2}$ & $d+\sigma^{2}$ \\
$\sigma^{*}$ & $\sigma$ & $\sigma$ & $\sigma$ \\
$\lambda^{*}$ & & $\frac{\lambda \eta_{2}}{\eta_{2}+1}$ & $\frac{\lambda p \eta_{1}}{\eta_{1}-1}+\frac{\lambda q \eta_{2}}{\eta_{2}+1}$ \\
$\eta_{1}^{*}$ & & & $\eta_{1}-1$ \\
$\eta_{2}^{*}$ & & $\eta_{2}+1$ & $\eta_{2}+1$ \\
$p^{*}$ & & & $\frac{p \eta_{1} \eta_{2}+p \eta_{1}}{\eta_{1} \eta_{2}+p \eta_{1}-q \eta_{2}}$ \\
\hline
\end{tabular}

overestimate the CoCos price for compressing jumps information into volatility. The result about overestimation for BS goes against the intuitive understanding that BS would underestimate the CoCos price without taking jump risk into consideration, which is due to the dual characteristics of CoCos. When $b \in(0,5.9)$, the small difference in trigger likelihood among these models leads to small difference in their
CoCos price. The trigger barrier level $b$ chosen as 8.5 is large enough for studying the difference in parameters' sensitivity analysis among these models.

Figure 2(a) shows that the CoCos price rises in all the models when $\lambda$ increases and the CoCos price in SNP and Kou increases slower than that in BS. The big difference in CoCos price between BS and the other two models and the small difference between Kou and SNP illustrate that negative jumps play far more important role than positive jumps in CoCos pricing, which shows practical value of SNP. Figure 2(b) shows the CoCos price increases in all the models when $\sigma$ in Kou increases. The increasing speed decreases in all the models for upper limit of the trigger probability. The increase of $\sigma$ makes $\sigma$ play more and more critical role in CoCos pricing, which alleviates the influence from jumps and makes the difference in CoCos price among these models decrease.

Figure 2(c) shows that the CoCos price decreases in all the models and the difference between BS and the other two models increases when $\eta_{1}$ increases. Since the average jump 

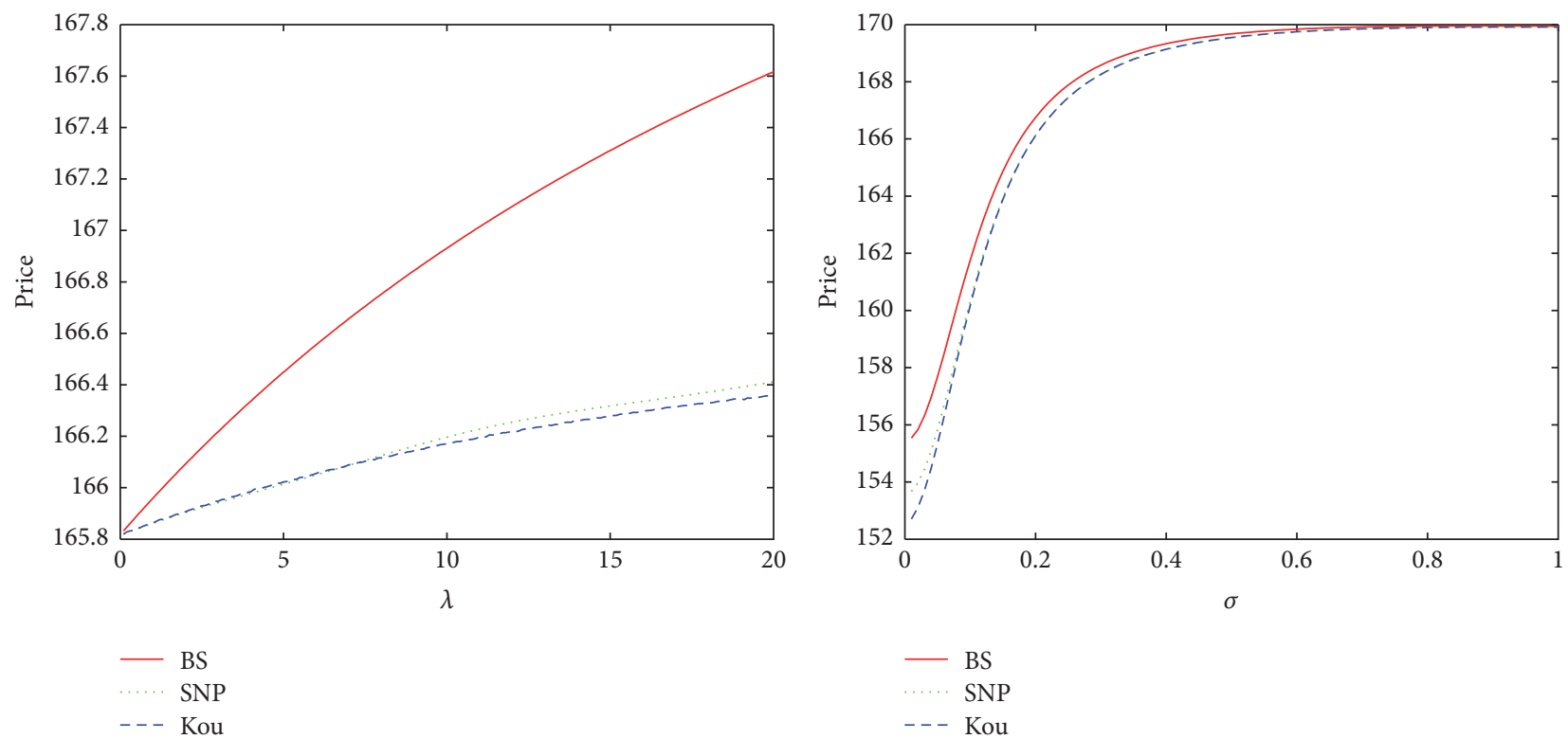

(a)

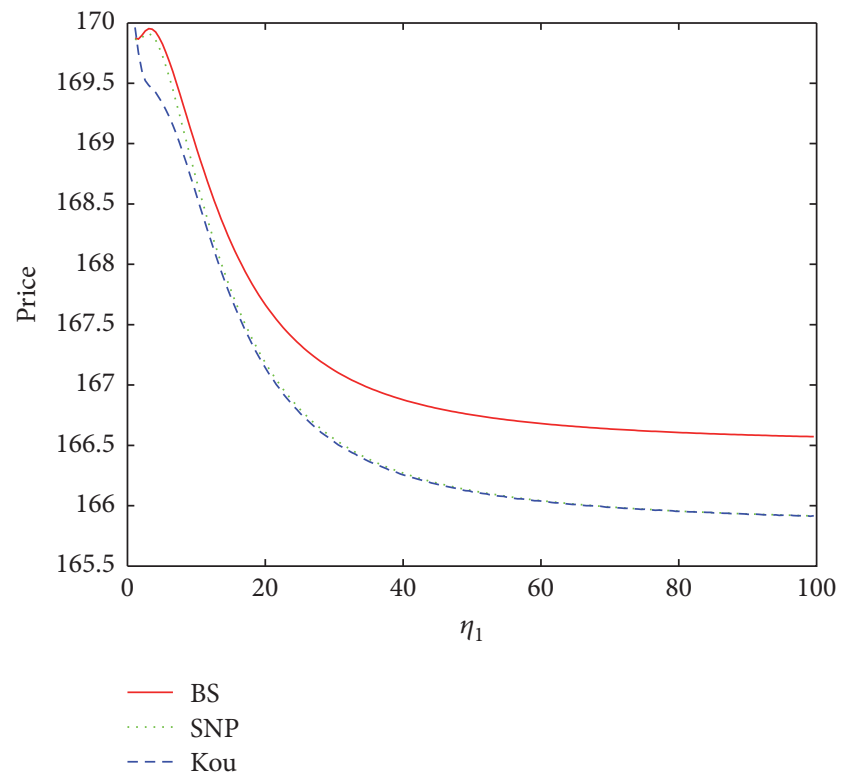

(c)

(b)

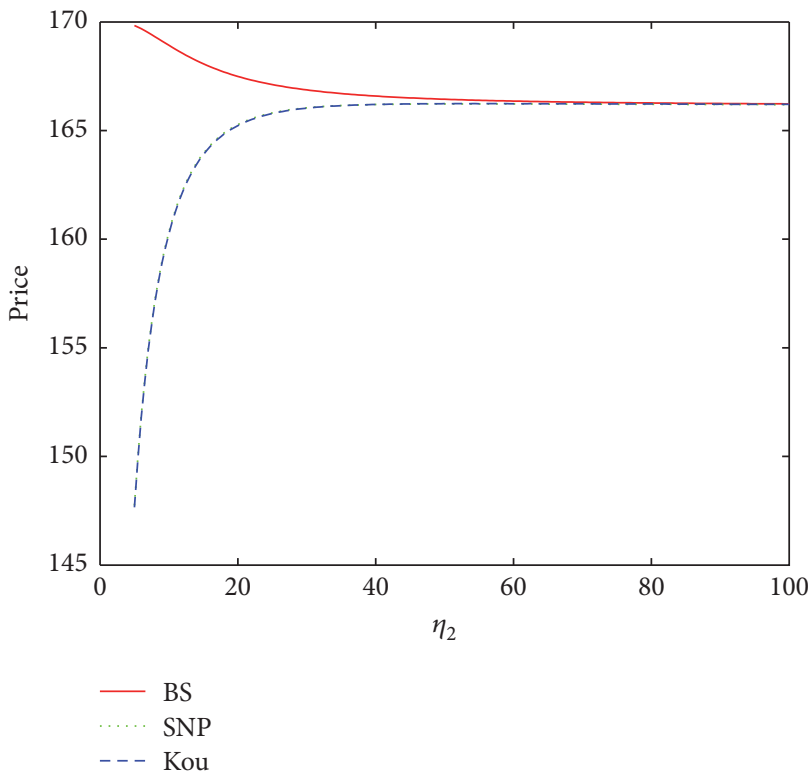

(d)

Figure 2: (a) The sensitivity of CoCos price to $\lambda$. (b) The sensitivity of CoCos price to $\sigma$. (c) The sensitivity of CoCos price to $\eta_{1}$. (d) The sensitivity of CoCos price to $\eta_{2}$.

size of positive jumps decreases, the effect of positive jumps becomes limited and negative jumps takes the leading role. Since the negative jumps have larger influence on the difference between BS and the other two models in CoCos price, the difference increases when $\eta_{1}$ increases. Figure 2(d) shows the CoCos price decreases in BS while that increases in Kou and SNP when $\eta_{2}$ increases. The difference between BS and the other two models in CoCos price decreases and the price in the models approximately converges to the same level. The decrease of average size of negative jumps weakens the influence of negative jumps, which reduces the difference in CoCos price between BS and the other two models.
From Figure 2(c), some difference exists in the CoCos price between SNP and Kou when average size of positive jumps is considerably larger than that of negative jumps, but this difference is not big when compared with the difference resulting from the influence of negative jumps. The comparison between Figures 2(c) and 2(d) also shows the more important role of negative jumps.

\section{Conclusion}

This paper develops a general Lévy framework for pricing CoCos with all-at-once conversion. The Lévy framework 
shows hybrid nature of CoCos intuitively and reduces the CoCos pricing problem to the first-passage time problem of trigger process. According to characteristics of new Lévy measure after the measure transform, three Lévy models driven by drifted Brownian motion, spectrally negative Lévy process, and double exponential jump diffusion process are proposed. These three Lévy models keep the form of the driving Lévy process unchanged under the measure transform, which sidesteps the difficulty that only rare forms of Lévy process have solved the first-passage time problem. These Lévy models provide closed-form expressions for CoCos price while two of them possess them up to Laplace transform, whose pricing results are given by combining with numerical Fourier inversion and Laplace inversion.

The numerical results show that the CoCos price is the trade-off between the opposite effects from debt part and equity part. Negative jumps play far more critical role than positive jumps in CoCos pricing. The Black-Scholes model compresses all the jumps information into volatility, which makes large difference in CoCos price between the BlackScholes model and the other two models. The model driven by spectrally negative Lévy process only compresses positive jumps information into volatility, which leads to small difference between the models driven by spectrally negative Lévy process and double exponential jump diffusion process. When the trigger barrier level is high, the difference between the Black-Scholes model and the other two models in CoCos price is significant. Without jumps characterization in trigger process, the Black-Scholes model would overestimate the CoCos price. The models driven by spectrally negative Lévy process and double exponential jump diffusion process would provide more accurate CoCos price by taking jumps phenomenon in financial market into consideration.

The Lévy models proposed in this paper can capture the short-run behaviour of trigger process. However, the longrun phenomenon such as the volatility clustering is not characterized. The stochastic volatility Lévy models and regime switching Lévy models, which can capture the long-run behaviour, deserve further study. Some special regime switching Lévy models have solved first-passage time problem and the next step can extend the Lévy models to regime switching Lévy models. Since different CoCos designs turn to different pricing models, the Lévy models for more complicated CoCos designs with characteristics such as multivariate trigger, the trigger based on moving average of easily observed and continuously updating indicators, and incorporation of contingent claims, such as buy-back option for converted shares and cancellable options for coupons, also need further study.

\section{Competing Interests}

The authors declare that they have no competing interests.

\section{Acknowledgments}

This research was supported by NSF of China (Grants nos. 71333014 and 71571007). JEL Classification is G12 G13 G21.

\section{References}

[1] M. Brennan and B. de Longevialle, "Potential \$1 trillion bank contingent capital-style issuance faces uncertain investor interest," Report, Standard \& Poors, 2010.

[2] M. J. Flannery, "No pain, no gain? Effecting market discipline via 'reverse convertible debentures,' SSRN Working Paper Series, 2003.

[3] M. J. Flannery, "Stabilizing large financial institutions with contingent capital certificates4," CAREFIN Research Paper 4, 2010.

[4] Squam Lake Working Group, An Expedited Resolution Mechanism for Distressed Financial Firms: Regulatory Hybrid Securities, Council on Foreign Relations Press, 2009.

[5] R. L. McDonald, "Contingent capital with a dual price trigger," Journal of Financial Stability, vol. 9, no. 2, pp. 230-241, 2013.

[6] S. Sundaresan and Z. Wang, "On the design of contingent capital with a market trigger," The Journal of Finance, vol. 70, no. 2, pp. 881-920, 2015.

[7] C. W. Calomiris and R. J. Herring, "How to design a contingent convertible debt requirement that helps solve our too-big-tofail problem," Journal of Applied Corporate Finance, vol. 25, no. 2, pp. 39-62, 2013.

[8] G. Pennacchi, T. Vermaelen, and C. C. P. Wolff, "Contingent capital: the case of COERCs," Journal of Financial and Quantitative Analysis, vol. 49, no. 3, pp. 541-574, 2014.

[9] J. M. Corcuera, J. De Spiegeleer, J. Fajardo, H. Jönsson, W. Schoutens, and A. Valdivia, "Close form pricing formulas for coupon cancellable cocos," Journal of Banking \& Finance, vol. 42, no. 1, pp. 339-351, 2014.

[10] P. Glasserman and B. Nouri, "Contingent capital with a capitalratio trigger," Management Science, vol. 58, no. 10, pp. 1816-1833, 2012.

[11] J. De Spiegeleer and W. Schoutens, "Pricing contingent convertibles: a derivatives approach," The Journal of Derivatives, vol. 20, no. 2, pp. 27-36, 2012.

[12] P. Cheridito and Z. Xu, "Pricing and hedging of coco's," SSRN Electronic Journal, 2015.

[13] G. Pennacchi, "A structural model of contingent bank capital," FRB of Cleveland Working Paper no. 10-04, 2010.

[14] C. Koziol and J. Lawrenz, "Contingent convertibles. Solving or seeding the next banking crisis?" Journal of Banking \& Finance, vol. 36, no. 1, pp. 90-104, 2012.

[15] T. Berg and C. Kaserer, "Does contingent capital induce excessive risk-taking?" Journal of Financial Intermediation, vol. 24, no. 3, pp. 356-385, 2015.

[16] J. Hilscher and A. Raviv, "Bank stability and market discipline: the effect of contingent capital on risk taking and default probability," Journal of Corporate Finance, vol. 29, pp. 542-560, 2014.

[17] C. P. Himmelberg, G. Sachs, and C. S. Tsyplakov, "Incentive effects and pricing of contingent capital," Working paper, 2014.

[18] B. Albul, D. M. Jaffee, and A. Tchistyi, "Contingent convertible bonds and capital structure decisions," Working paper, 2015.

[19] A. Metzler and R. M. Reesor, "Valuation and analysis of zerocoupon contingent capital bonds," Mathematics and Financial Economics, vol. 9, no. 2, pp. 85-109, 2015.

[20] S. Wilkens and N. Bethke, "Contingent convertible (CoCo) bonds: a first empirical assessment of selected pricing models," Financial Analysts Journal, vol. 70, no. 2, pp. 59-77, 2014. 
[21] J. Manuel Corcuera, J. de Spiegeleer, A. Ferreiro-Castilla, A. E. Kyprianou, D. B. Madan, and W. Schoutens, "Pricing of contingent convertibles under smile conform models," The Journal of Credit Risk, vol. 9, no. 3, pp. 121-140, 2013.

[22] J. M. Corcuera and A. Valdivia, "Pricing cocos with a market trigger," in Stochastics of Environmental and Financial Economics, pp. 179-209, Springer, Berlin, Germany, 2016.

[23] P. Tankov, Financial Modelling with Jump Processes, vol. 2, CRC Press, Boca Raton, Fla, USA, 2003.

[24] A. Kyprianou, Introductory Lectures on Fluctuations of Lévy Processes with Applications, Springer Science \& Business Media, 2006.

[25] D. Applebaum, Lévy Processes and Stochastic Calculus, Cambridge University Press, 2009.

[26] S. G. Kou and H. Wang, "First passage times of a jump diffusion process," Advances in Applied Probability, vol. 35, no. 2, pp. 504531, 2003.

[27] L. C. Rogers, "Evaluating first-passage probabilities for spectrally one-sided lévy processes," Journal of Applied Probability, vol. 37, no. 4, pp. 1173-1180, 2000. 


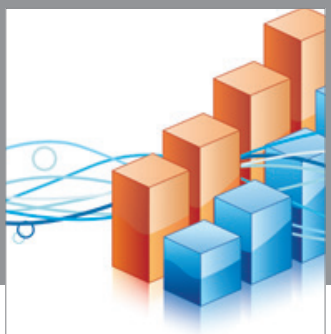

Advances in

Operations Research

vatem alat4

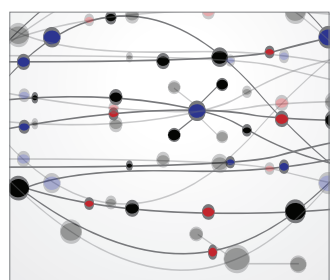

\section{The Scientific} World Journal
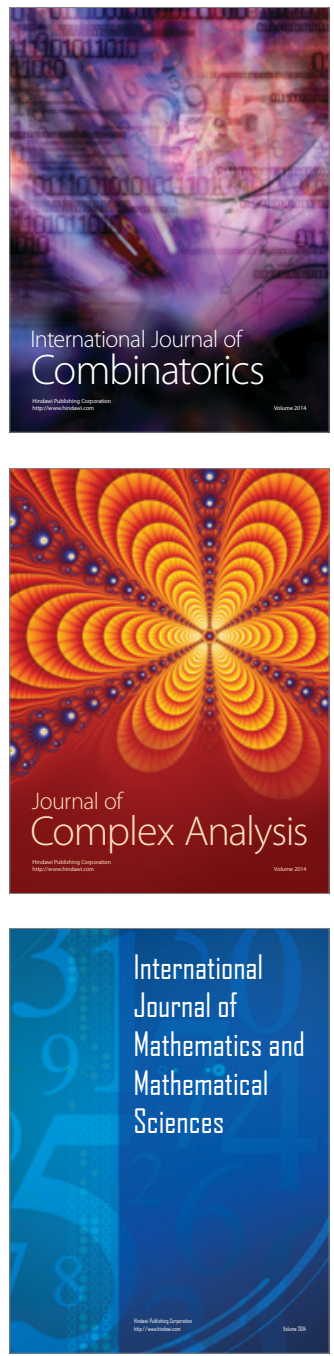
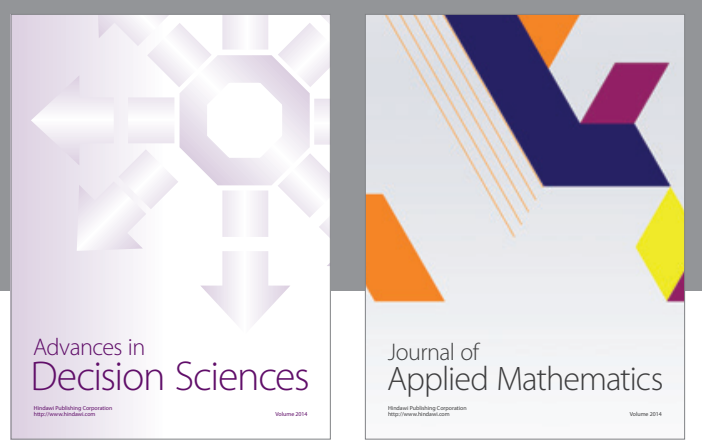

Algebra

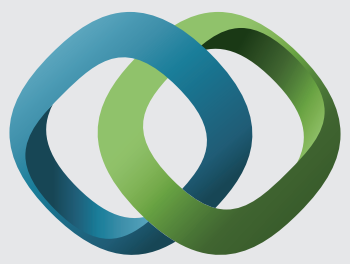

\section{Hindawi}

Submit your manuscripts at

https://www.hindawi.com
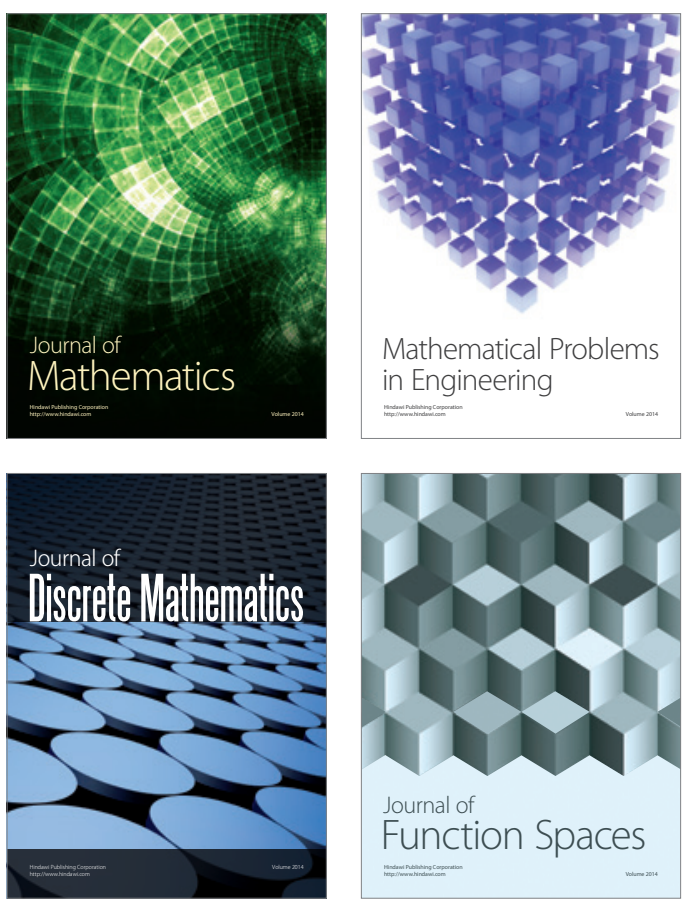

Mathematical Problems in Engineering
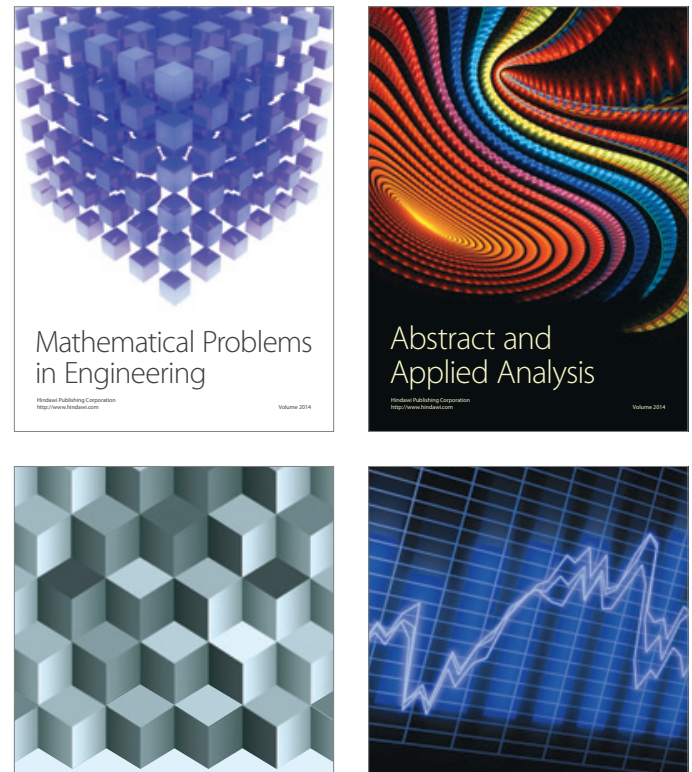

Journal of

Function Spaces

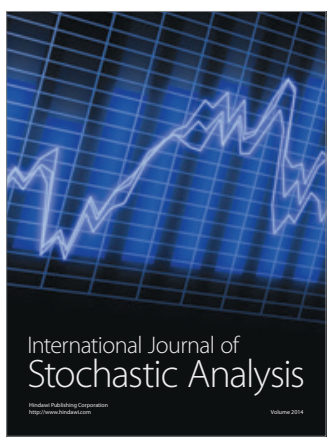

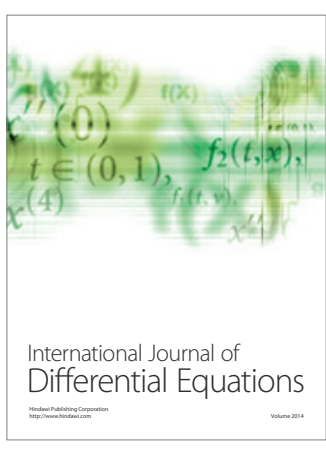
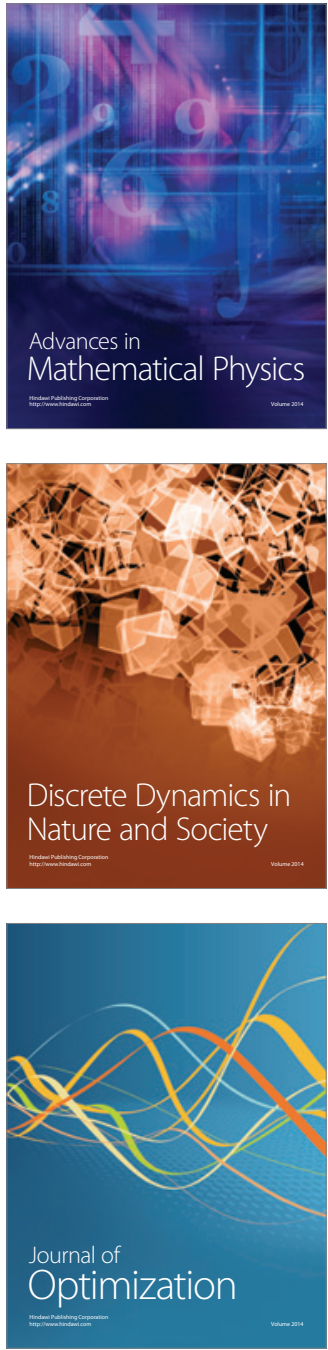